\title{
Zwischen Recht auf Stadt und Creative City - das Projekt „Alm DIY“6
}

\author{
Fabian Sandholzer
}

\section{$1 \quad$ Einleitung}

In den vergangenen Jahren ist eine Fülle lose verwandter Aktivitäten in den Fokus wissenschaftlicher und planerischer Diskurse gerückt. Unter Labels wie „,informeller Urbanismus“, „tactical urbanism“ oder „subversiver Urbanismus“ wird eine Reihe von städtischen Praktiken zusammengefasst. Ihren Niederschlag finden sie meist in Bespielungen oder Aneignungen des öffentlichen Raumes oder in Zwischennutzungen von leer stehenden Gebäuden/Brachflächen (vgl. Iveson 2013: 941). Willinger (2014: 149) subsumiert ein breites Spektrum an Protagonisten als „,informelle Akteure der Stadtentwicklung“: von Energiegenossenschaften, Sportvereinen und Bürgerstiftungen bis hin zu Guerilla Gardening, wildem Skaten oder BMX. Diese Phänomene und deren Beitrag zum städtischen Leben im Quartier, können einen vielversprechenden Ausgangspunkt für demokratischere Städte und das Hinterfragen von konventionellen Top-down-Planungsansätzen bilden.

Der folgende Beitrag beschäftigt sich deshalb ausführlich mit einem Do-ityourself-Skateboard-Projekt namens „Alm DIY“, das als ungenehmigte, temporäre Nutzung einer brachliegenden Fläche inmitten eines der größten Stadtentwicklungsprojekte Wiens begann. Ausgehend vom „Recht auf Stadt“, das der französische Philosoph Henri Lefebvre postulierte, stellt sich die Frage, inwieweit dieses Projekt eine demokratische Form der Mitgestaltung und Rückgewinnung der Stadt darstellt. Gleichzeitig werden sich Stadtverwaltungen und -entwickler*innen immer mehr des Potenzials bewusst, welches diese Initiativen mit sich bringen können. Besonders für kulturell geprägte Entwicklungsstrategien wie die Creative City (vgl. Florida 2002) scheinen diese Aktivitäten einen besonderen Reiz auszuüben. Neben dem theoretischen Hintergrund greift dieser Beitrag auch auf die im Rahmen der Masterarbeit des Autors erhobene Empirie ${ }^{1}$ zurück.

1 Im Jahr 2016 wurden Interviews mit „Alm DIY“ (i. F. Interview 1), „Nordbahnhofgelände für Alle“ (i. F. Interview 2), DIin Jutta Kleedorfer von „einfach - mehrfach“ (i. F. Interview 


\section{Skateboarden und Stadt}

Schon vergleichsweise lange sind Skateboarder*innen in vielen Städten präsent und zogen auch immer wieder das Interesse der Stadtforschung auf sich (z. B. Borden 2001; Chiu 2009; Howell 2008; Peters 2016; Schweer 2014). Die verschiedenen Praktiken des Skateboardens werden im Folgenden kurz dargelegt, um anhand dessen die unterschiedlichen Zusammenhänge und Wechselwirkungen mit dem Stadtraum zu verdeutlichen. Dies soll als Grundlage für die späteren Ausführungen zum betrachteten Fallbeispiel „Alm DIY“ dienen.

\subsection{Street-Skaten}

Das Street-Skaten spielt sich in der Wildnis der Stadt ab. Laut Peters (2016), ähnlich auch bei Borden (2001) und Vivoni (2013), gibt es im Street-Skaten zwei zentrale Aktivitäten: zum einen das Umherschweifen auf Skateboards durch die

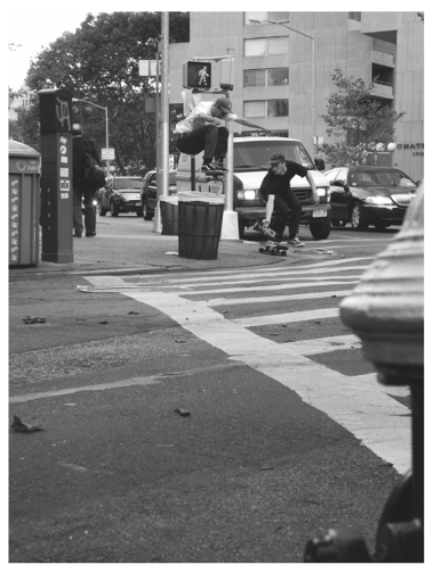

Abbildung 1: $\quad$ Ein Skateboarder überwindet einen Müllkübel mit einem „Ollie“. Grobkörniger Asphalt, Fußgänger- und Autoverkehr erschweren das Ausführen des Manövers, erhöhen aber zusammen mit dem Straßenbild von New York City den Wert der Aufnahme des nebenan fahrenden Filmers.

Quelle: F. Sandholzer, 2013

Kleedorfer) und DI Martin Haas von der WSE Wiener Standortentwicklung GmbH (i. F. Interview Haas) geführt. 
Stadt und zum anderen die spielerische Raumverhandlung eines konkreten „Skatespots". Alltägliche Objekte erhalten durch unterschiedliche Eigenschaften einen speziellen Status im Blickwinkel der Skateboarder und werden als „Skatespots“ entdeckt. Mauern, Bänke, Geländer oder Treppenstufen werden in der Skate-Logik nicht in der von der Planerin bzw. dem Planer oder der Architektin bzw. dem Architekten intendierten Funktion gelesen (vgl. Abb. 1) und stattdessen für die Durchführung von Skateboard-Tricks zweckentfremdet. Im Vordergrund steht dabei nicht immer nur die beste materielle Beschaffenheit einer Architektur, um darauf Tricks zu vollziehen. Ästhetische und symbolische Eigenschaften oder die geografische Lage können - besonders in Hinblick auf eine mediale Verwertung - ebenso entscheidend für die Popularität und den Stellenwert eines Spots sein (vgl. Peters 2016: 143).

\subsection{Park-Skaten}

Mit den Skateparks und deren Variationen gibt es gebaute Sonderräume, welche explizit zum Zweck des Skateboardfahrens errichtet worden sind. Deren Gestaltung reicht von der Imitation der städtischen „Natur“ (sogenannten Streetplazas) bis hin zu leeren Swimmingpools nachempfundenen Landschaften (sogenannten Bowls oder Pools).

„Skateboarders Won“ betitelte die New York Times einen Artikel, der die zunehmende Anzahl von Skateparks und die ins Positive umgeschlagene Stimmung städtischer Verwaltungen gegenüber deren Errichtung behandelt (vgl. Ihaza 2018). Gleichzeitig verdeutlicht das im selben Artikel auftauchende Zitat „Skate parks have always been designed to contain an activity that is about roaming, and often dangerous, or at least unlawful trespassing " (Barrow zit. nach Ihaza 2018: o. S.) die andere Seite des vermeintlichen Erfolges. Stadtverwaltungen sehen hier eine Möglichkeit, die Praxis des Street-Skatens zu disziplinieren und sie aus dem öffentlichen Raum in gemaßregelte Sonderräume umzulenken. Zusammen mit einem repressiven Vorgehen gegenüber Street-Skaten führen sie so zu einer Normierung, Disziplinierung und Segregation des Skateboardfahrens (vgl. Peters 2016: 152 f.).

\subsection{DIY-Skaten}

Schon vor dem gegenwärtigen Boom des Selbermachens hatte das DIY-Skaten innerhalb der Skateboardwelt seinen Platz. Es unterscheidet sich von anderen Skateboard-Praktiken durch die eigenständige Veränderung der gebauten Umwelt und setzt somit zusätzlich handwerkliches Können voraus (vgl. Abb. 2). Bereits mini- 
male Eingriffe wie etwa das Ausbessern eines Spalts mit Blitzbeton können aus einem nicht befahrbaren Objekt einen Skatespot schaffen. Oftmals entstehen aber auch ganze Skatestrukturen. In der Regel sind diese weder legal errichtet noch professionell erbaut und liegen auf brachliegenden Flächen (vgl. Schweer 2014: 51).

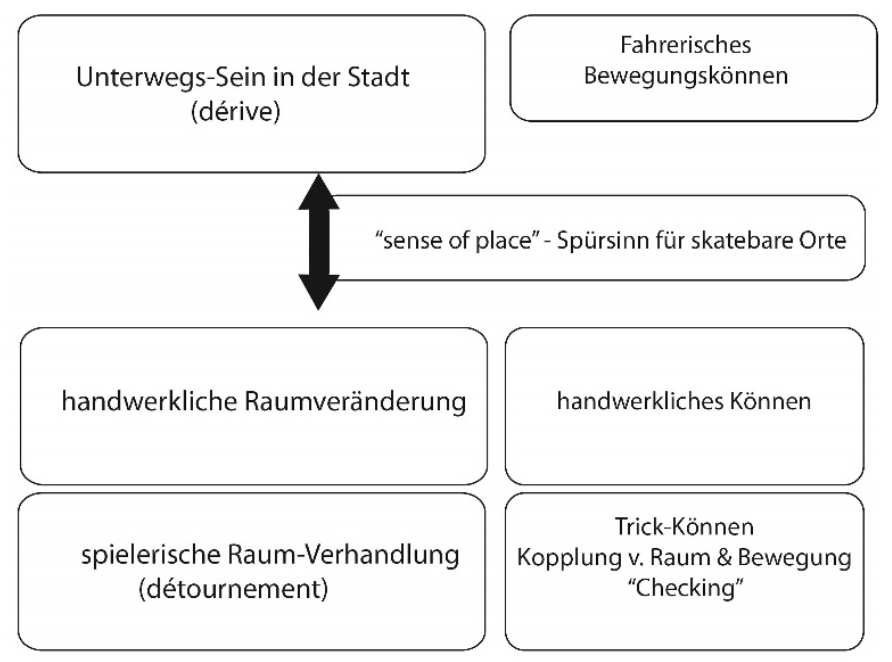

Abbildung 2: Komponenten der Praktik des DIY-Skatens

Quelle: Peters (2016: 159)

Als emblematisches Beispiel des DIY-Skatens gilt der unterhalb der Burnside Bridge in Portland, USA, errichtete Burnside Spot. Im Sommer 1990 startete eine Gruppe lokaler Skateboarder*innen ihr Projekt auf einem Parkplatz eines verlassenen Hotels unterhalb der Brücke. Anfangs wurde zusammen mit Obdachlosen begonnen, eine Schräge aus Beton zu bauen. Seitdem wurden nach und nach Rampen hinzugefügt, wieder abgerissen und modifiziert. Die Stadtbehörden ignorierten den selbst erbauten Skatepark zunächst, drohten dann mit dem Abriss, bevor sie ihn schlussendlich offiziell genehmigten (vgl. Borden 2001: 76). Mit ein Grund für die positive Stimmung der städtischen Behörden gegenüber dem Projekt war ein beobachteter Rückgang gewisser Formen von Kriminalität in der Nachbarschaft sowie die Aufwertung der zuvor vor allem von Drogenkonsument*innen, Alkoholkranken und Prostituierten genutzten Fläche (vgl. Lombard 2010: 485 f.).

Peters (2016) sieht im DIY-Skaten eine starke Betonung der Eigenwilligkeit und Eigensinnigkeit des Skateboardfahrens und baut den Gedanken aus, indem er es als Rückführung des Skatens aus dem kulturellen Mainstream wieder hin zur 
Subkultur sieht. Er beschreibt das DIY-Skaten als Reaktion auf die zunehmende Kommerzialisierung (vgl. Schweer 2014) und das dadurch ausgelöste Bedürfnis nach „Nahraum-Erfahrungen und eigentätiger Ästhetisierung der persönlichen Nahwelt" (Peters 2016: 155 f.). Da ein DIY Spot-immer auch Arbeit im Sinne des Bauprozesses an einem selbst und im Kollektiv ist, erschafft er gemeinsame, an den Ort geknüpfte und von diesem ausgestrahlte Identität. Schweer betrachtet DIY-Spots auch deswegen als Heterotopien im Sinne Foucaults, da sich die Skateboarder „Räume schaffen, welche ihren eigenlogischen Bedürfnissen entsprechen und die in Abgrenzung zur gesellschaftlichen Umwelt eigene Werte und Handlungsparameter setzen“" (Schweer 2014: 55).

Sowohl DIY-Spots als auch reguläre Skateparks, -hallen und -plazas sind speziell zum Skaten gebaute Strukturen. Ihre Unterschiedlichkeit hebt Vivoni (2013) anhand der Beispiele des Burnside Parks als DIY-Spot und eines vom Schuhersteller Vans in einem Einkaufszentrum errichteten Skateparks hervor:

"The first is grassroots, illegal, and on the fly while the second is air conditioned, forprofit, and highly regulated. Furthermore, each skatepark claims divergent social settings and reproduces dissimilar social relations. The Burnside Project is founded on do-it-yourself anti-authoritarian camaraderie while the Vans Skatepark is set in elite consumption through membership fees and enforced rules and regulations "(Vivoni 2013: 52).

Im Spektrum von zweckgebauten Spots und Parks gibt es eine Reihe von verschiedenen Ausprägungen. DIY-Spots und -Parks müssen auch nicht immer illegal sein; ein legales Beispiel ist eine auf dem Gelände des Tempelhofer Feldes in Berlin realisierte Skateskulptur. Die Errichtung dieser geschah im Rahmen einer partizipativen Baustelle und unter Verwendung von aus dem Abriss des Palastes der Republik stammenden Granitplatten (vgl. Freivogelheit GmbH 2012). Die meisten DIY-Spots sind allerdings durch ihre Illegalität ein temporäres Phänomen, doch macht das ständige Bauen neuer Spots einen Teil der Attraktivität aus. Der in der Szene für seinen Einsatz für DIY-Spots bekannte schwedische Skater Pontus Alv meint dazu:

„Well we have been building DIY spots here for about ten years and we have seen our spots go up and come down. It always hurts and it always sucks, but it is a part of the game. The spots are temporary and always in progress, these spots are organic and we all know they won't last forever. But this is what makes them so good, it fires up the sessions and you push it all a bit extra 'cause you never know when it will all be gone " (Irvine 2013: o. S.). 


\section{Das Projekt „Alm DIY“}

Entlang der in Kapitel 2.3 beschriebenen Praxis des DIY-Skatens entwickelte sich im 2. Wiener Gemeindebezirk das sogenannte Alm-DIY-Projekt.

Auf dem Areal des ehemaligen Nordbahnhofs begann 2014 ein aus einer Kerngruppe von sechs bis sieben Personen bestehendes Kollektiv damit, erste Rampen in Eigenregie zu betonieren. Die dabei bebaute brachliegende Fläche auf dem ehemaligen Bahnhofsgelände liegt inmitten eines der größten Stadtentwicklungsgebiete Wiens. Etwa die Hälfte des gesamten Gebiets ist bereits bebaut; für den nordwestlichen Teil wurde 2014 ein neues städtebauliches Leitbild entwickelt. Dieses sieht ein großzügiges Freihalten der Mitte als naturnah ausgestalteten Grünraum und eine Konzentration der vielseitig genutzten Randbebauung vor (vgl. Magistratsabteilung 21 2015: 17). Es wurde in einem Partizipationsprozess erarbeitet und sieht in der freien Mitte auch die Möglichkeit vor, dass ,sinnvolle Zwischennutzung[en; F.S.] von Teilen des Areals für die NutzerInnen des Stadtteils“ (ebd.: 31) entstehen können. Die von der Alm-DIY-Gruppierung genutzte Fläche liegt in dieser ,freien Mitte“ und somit auf einem Areal, das zukünftig gar nicht bebaut, sondern höchstens landschaftsgärtnerisch gestaltet werden soll - wobei hier ebenfalls im Leitbild erwähnt wird, dass die „freie Mitte“ naturnah und weitgehend unverändert erhalten bleiben soll (vgl. ebd.: 40).

Als keine offensichtlichen Probleme mit der Bebauung und Nutzung auftauchten, wurde der Platz nach und nach ausgebaut. Nur wenige Wochen darauf wurden die Betonrampen allerdings im Auftrag des Grundstücksbesitzers, der Österreichischen Bundesbahnen (ÖBB), abgerissen. Nachdem der Abbruch in Berichten lokaler Medien thematisiert worden war, signalisierten die ÖBB Gesprächsbereitschaft bezüglich einer etwaige Zwischennutzung auf der Fläche. Aus dem Umfeld der Erbauer*innen wurde daraufhin der „Verein zur Förderung von Skateboard D.I.Y. Projekten“" gegründet und ein vorerst auf ein Jahr befristeter Vertrag zwischen Verein und Grundstückseigentümer ermöglichte einen zweiten Anlauf von „Alm DIY“. Bei den Verhandlungen zwischen Verein und Grundstückseigentümer war auch das auf Mehrfach- und Zwischennutzungen spezialisierte Projekt ,einfach - mehrfach“ der Stadt Wien beteiligt. Neben der beratenden Unterstützung konnte auch eine finanzielle Förderung vonseiten der Stadt erreicht werden (vgl. Interview Kleedorfer). Das Kollektiv begann nun wiederum mit dem Betonieren von Rampen und erweiterte das Areal um Gemeinschaftsgärten, Fitnessgeräte und einen Aufenthaltsbereich (vgl. Abb. 3). Zusätzlich dazu wurden die Betonrampen auch zur Erstellung von Street-Art genutzt. Neben den Skateboarder*innen, die sich aus der gesamten Stadt, teilweise auch aus ganz Europa und der Welt, auf den Weg zu den Rampen machten, entdeckte auch die Nachbarschaft den Platz für sich. Die beim Projekt involvierten Anrainer*innen hingegen 
kamen primär nicht zum Skaten, sondern waren eher bei den Gemüsebeeten engagiert oder nutzten den Platz, um mit ihren Kindern Fußball zu spielen. Eine weitere Nutzer*innengruppe waren etwas ältere Kinder und Jugendliche, welche den Platz ebenfalls für sich entdeckt hatten und ihn als Abenteuerspielplatz oder zum Tretrollerfahren nutzten (vgl. Interview 2). Auch wenn die Schaffung eines in Eigenregie und durch Selbstverwaltung gestalteten Platzes eines der Hauptmotive für die Errichtung der „DIY-Alm“ war, ermöglichte die offene Gestaltung eine vielseitige Bespielung und schuf so einen Ort, welcher gerade nicht als exklusiv und kollektiv geschlossen zu bezeichnen ist, wie es teilweise DIY-Spots zugeschrieben wird (vgl. Peters 2016: 157). Dies kommt, neben der Einbeziehung der Nachbarschaft aus dem Quartier und der offenen Gestaltung, auch in einer Eigenbeschreibung des Projekts zum Ausdruck: „Es ist ein selbstverwalteter Platz, der in Eigeninitiative bespielt wird, so vielfältig wie möglich “ (Interview 1).

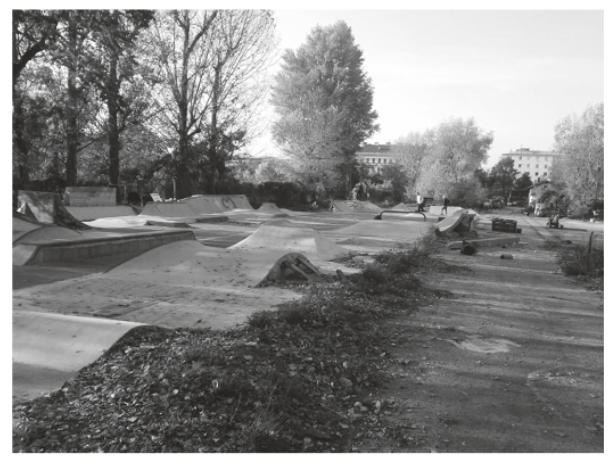

Abbildung 3: Alm DIY“ auf dem Gelände des ehemaligen Nordbahnhofs. Das Foto zeigt die Betonrampen des zweiten Aufbaus.

Foto: F. Sandholzer, 2015

Neuerliches Konfrontationspotenzial kam vor allem nach dem Ende des auf ein Jahr befristeten Mietvertrages auf. Der ursprünglich bei Abschluss des Vertrages noch antizipierte Übergang des Grundstückes an die Stadt Wien wurde nicht vollzogen und so kam es wiederum zu Verhandlungen über die Zukunft des Projekts. Eine kurzfristige Verlängerung des Vertrages für einen Monat konnte zwar relativ rasch erreicht werden; schlussendlich wurde eine Verlängerung aber vonseiten der ÖBB abgelehnt. Der damalige Bezirksvorstand zeigte gegenüber den Aktivist*innen rund um den Verein eine ablehnende Haltung, während andere städtische Stellen wie „einfach - mehrfach“ oder der zuständige Projektsprecher der Wiener Baudirektion eine positive Einstellung dazu hatten (vgl. Grabner 2016). Auch der 
paradoxe Umstand, dass im städtebaulichen Leitbild für das derzeit entstehende Nordbahnhofviertel explizit die Möglichkeit zukünftiger Zwischennutzungen auf der Fläche des Alm-DIY-Projekts vorgesehen sind, änderte nichts am vorzeitigen Ende des Vertrages.

\section{$4 \quad$ Ein Beispiel für die Ausübung des Rechts auf Stadt?}

In „Le droit à la ville“ umreißt Lefebvre das Recht auf Stadt als ein Recht auf Nichtausschluss von den Qualitäten der urbanisierten Gesellschaft. Ausgehend von einer Kritik der Verhältnisse der fordistisch-kapitalistischen Stadt fordert er das Recht auf Stadt ein - vor allem für jene, die dem verordneten Alltag in der Stadt ausgesetzt sind (vgl. Gebhardt \& Holm 2011: 7 f.). Seit den 1990er-Jahren hat der Begriff eine Renaissance erfahren. Verschiedenste Gruppierungen haben die Arbeiten von Lefebvres wiederentdeckt, deren Relevanz vor dem Hintergrund neuer Konflikte durch die Neoliberalisierung des Städtischen gestiegen ist (Mullis 2013: 61).

Grundlegend ist die Errichtung des DIY-Spots auf dem Nordbahnhofgelände unter dem Aspekt einer kollektiven Wiederaneignung des städtischen Raumes zu sehen und somit dementsprechend an eine Kernthese des „Recht auf Stadt“-Begriffs angebunden (vgl. Gebhardt \& Holm 2011: 8). In der Gemeinschaft wurde die Brachfläche in ihrer physischen Gestalt verändert und ihr ein am Gebrauchswert der Stadt orientiertes Leben eingehaucht. Die Bestrebung, den Tauschwert und somit den Marktwert der Fläche nicht zu schmälern, stand in Konflikt zu dem von den Nutzern aktivierten Gebrauchswert. Somit ist hier, wie auch fast immer, das Recht auf Stadt ein Ringen um eine Verbesserung der Stellung von Bewohner*innen der Stadt gegenüber von Eigentumsrechten von Grundstücksbesitzer*innen (vgl. Purcell 2014: 142). Die Auseinandersetzungen um diesen Konflikt regten wiederum Debatten über zukünftige städtische Entwicklungen und die mit der Stadt in Wechselwirkung stehenden sozialen Verhältnisse und Praktiken an, etwa auf formaler Ebene in Bezirksratssitzungen oder auf informeller Ebene in Nachbarschaftsgesprächen.

Als weiterer zentraler Aspekt des Rechts auf Stadt wurde die Fokussierung auf die Bewohner*innen der Stadt beim Alm-DIY-Projekt am Standort Nordbahnhof verwirklicht. Im Gegensatz zu formalen Kriterien, wie etwa einer Staatsbürgerschaft, sind es die alltäglichen Erfahrungen der Skateboarder*innen in der Stadt, welche sie dazu befähigen, die Stadt zu gestalten. , [...] it is the everyday experience of inhabiting the city that entitles one to a right to the city " (Purcell 2014: 142).

Die Skateboarder*innen berufen sich hier nicht wie andere Bewegungen und Initiativen explizit auf den Slogan „Recht auf Stadt“. Sie folgen eher der bei 
Schweer (2014: 37) erwähnten Logik: ,, Skateboarder haben keinen >Masterplan zur Veränderung der Stadt oder der Gesellschaft, sie setzen im Kleinen, Konkreten und Praktikablen an. " Trotzdem verfügt das Projekt über ein nicht zu unterschätzendes alternatives Potenzial. Ähnlich wie bei den von Iveson (2013) genannten Fallbeispielen wurde auf dem Alm-DIY-Gelände eine „city within the city“ geschaffen. Damit ist gemeint, dass durch die Aneignung des Raumes auch eine andere Form der Autorität kreiert wurde. Das alternative Potenzial einer Brachfläche wurde - gemäß Lefebvres Ausspruch „,beneath the road, the beach“ - realisiert, indem die Akteur*innen das Recht auf Stadt einforderten und die dominanten Autoritäten der Stadt, wie Eigentümer*innen oder die Polizei, zumindest anfangs ignorierten. Vor dem Erbauen der Rampen wurde das Grundstück nicht gekauft, um keine Erlaubnis verhandelt oder um diese gefragt - vielmehr implizierte das Handeln der Skater*innen, dass sie als Bewohner*innen der Stadt genauso dazu befähigt seien, dieses zu gestalten.

Der zweite wichtige Punkt, den Iveson (2013: 946) für eine Politisierung von informellen Urbanismen sieht, ist, in Konfrontation zu den dominanten Machtstrukturen der Stadt zu gehen und diese auch ein Stück weit zu inszenieren. Dies geschah im Falle von „Alm DIY“ bis zu einem gewissen Grad. Die finanzielle und auch die bei den Verhandlungen mit dem Grundstückseigentümer geleistete Unterstützung vonseiten der Stadt, die auf die anfängliche Konfrontation durch den Abriss folgte, signalisiert bereits, dass aus einer Konfrontationssituation zu einem gewissen Maß eine Kooperation wurde. Schweer (2014: 55) beschreibt diese Legalisierung des angeeigneten Raumes von DIY-Spots als Konsensseite:
„Hier wird der angeeignete Raum legalisiert, also in die Ordnung aufgenommen, und kann im Urban Governance Kontext als Vorzeigeobjekt eigenverantwortlicher und unternehmerisch denkender junger Menschen fungieren [...]. Dabei wird der Selbst- verwaltungsanspruch der Skateboarder aufgegriffen und die Verantwortung für die Anlage auf die Skateboarder übertragen, welche sihren Platz selbst verwalten (self policing) und potenziell zum urbanen Ordnungsfaktor werden. "

Setzt man die Geschehnisse gegen Ende des Vertrages (vgl. Kap. 3) in Bezug zu den von Iveson dargestellten Zusammenhängen zwischen DIY-Urbanismen und dem Recht auf Stadt, stellt sich die Frage, inwiefern das Einzelprojekt „Alm DIY“ seine Strahlkraft bzw. die Durchsetzung eines Rechts auf Stadt befördern konnte. Iveson meint dazu:

“[...] 'appropriating' urban space for unintended uses does not in itself give birth to a new kind of city. There is no guarantee that the proliferation of DIY experiments with appropriation and alternative uses of urban space will coalesce into a wider politics of the city. However, the prospects for such a politics do exist and must be teased out if small-scale projects are to coalesce into large-scale change. I argue here 
that building a politics to connect the practices is a matter of both appropriation and political subjectivization, in which practitioners make themselves parties to a disagreement over the forms of authority that produce urban space" (Iveson 2013: 942).

Beim Alm-DIY-Projekt wurde dieses Potenzial auf verschiedene Weise hervorgerufen. Zum einen kam es durch die Benutzung des Platzes durch verschiedene Gruppen und Initiativen (Skateboarder*innen, Gärtner*innen etc.) zu einer Wirkung über die ursprüngliche Gruppe hinaus. In der Diskussion rund um das Ende des Mietvertrages wurden auch grundlegende Fragen nach der Gestaltung von Freiräumen in der Stadt gestellt und artikuliert. Besonderer Ausdruck davon ist das am 18.3.2016 abgehaltene Fest für Freiräume, bei dem der selbst verwaltete Platz noch einmal von vielen unterschiedlichen Gruppen gefeiert und für dessen Erhalt gekämpft wurde. Dabei wurde nicht nur für den Fortbestand des Platzes demonstriert, sondern es wurden auch etablierte Formen der Autorität, nach denen Freiräume nicht direkt von den Bewohner*innen der Stadt gestaltet werden, infrage gestellt. Somit kann das Projekt „Alm DIY“ neben die von Iveson aufgeführten Beispiele gestellt werden, da es ebenfalls dem Prinzip ,, assert new forms of authority in the city based on the equality of urban inhabitants " (Iveson 2013: 941) folgt.

Trotzdem lässt sich die Wirkung des Projekts mit Margit Mayers Arbeiten zu Lefebvre relativieren. Grob unterscheidet Mayer - ähnlich wie Marcuse (2009) zwei verschiedene Lager, welche gegen die neoliberale Stadt aufbegehren. Auf der einen Seite sind es (sub)kulturelle Aktivist*innen aus dem künstlerischen oder alternativen Milieu, auf der anderen Seite von der Austeritätspolitik der neoliberalen Städte betroffene Gruppen (Mayer 2013: 162 f.). Das Projekt „Alm DIY“ ist hier vor allem ersterer Gruppe zuzuordnen und kann die geforderte Verbindung beider Bewegungsgruppen nur bedingt herstellen. Zudem stellt der Kampf dieser Akteur*innen nicht wirklich eine Bedrohung der Herrschafts- und Ausbeutungsstrukturen des globalen neoliberalen Systems dar (Mayer 2011: 71). Ein Grund ist auch die Andockmöglichkeit für Standortpolitik und Vermarktungsstrategien zur Attraktion von Kreativen, Tourist*innen und Investor*innen (Mayer 2013: 162), auf welche in Kapitel 6 noch genauer eingegangen werden soll.

\section{Neuer Standort in St. Marx}

Nach dem Ende des Alm-DIY-Projekts auf dem Nordbahnhofgelände hatte der Verein bereits ein neues Areal in Aussicht, um dort ein weiteres DIY-Projekt zu verwirklichen. Anders als zuvor wurde das Kollektiv rund um den Verein dieses Mal gezielt durch die Wiener Standortentwicklung (WSE) für eine Zwischennutzung im 3. Wiener Gemeindebezirk angeworben (vgl. Interview Haas). Die WSE 
ist wiederum Teil der Wien Holding und somit im Eigentum der Stadt Wien. Sie ist mit der Entwicklung des Areals des ehemaligen städtischen Schlachthofs St. Marx beschäftigt, einem Prozess, der seit 2010 unter dem Label neu marx vorangetrieben wird. Bereits 2004 wurde mit der Fertigstellung des T-Centers ein erster Grundstein für eine Entwicklung in Richtung Wissens-, Technologie- und Medienstandort gelegt. Neben Gewerbe- und Dienstleistungsnutzungen entstanden aber auch Wohnquartiere und so sind es im Gebiet neu marx aktuell knapp 7.000 Menschen, die hier arbeiten und leben (vgl. Raumposition 2016: 34). Insgesamt zeigen der Fokus auf die Kreativindustrie und die Planungsunterlagen eine Entwicklung des Standorts im Sinne einer an die Creative City (vgl. Florida 2002) angelehnten Strategie (vgl. Abb. 4) und der bei Mould (2015) als deren physische Manifestation beschriebenen Media Cities bzw. Cultural Quarters. So sind in den Immobilien des „Media Quarter Marx“ bereits zahlreiche Mieter*innen aus der Medienbranche bzw. der ,creative economy“ einquartiert, darunter etwa eine Zeitungsredaktion und TV-Studios (vgl. WSE 2016: 4).

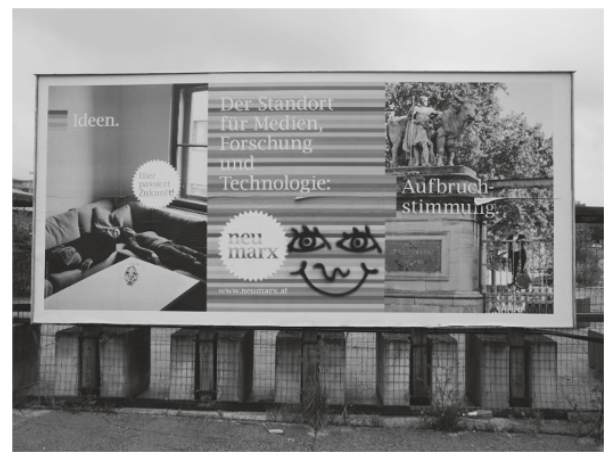

Abbildung 4: Werbeplakat für den Standort neu marx

Foto: F. Sandholzer, 2016

Auf der bisher noch brachliegenden Fläche der Karl-Farkas-Gasse 1 wurden verschiedene Zwischennutzungen angesiedelt. U. a. geschah dies, um die Fläche bekannt zu machen und ihr Image aufzuwerten - besonders im Milieu der für die spätere Nutzung angedachten Start-up- und Kreativszene. In der Bewerbung dieser steckt auch einer der Hauptgründe für die Anwerbung der Skateboarder*innen als Zwischennutzer*innen (vgl. Interview Haas). Wie schon bei Schweer (2014: 106) hervorgehoben, sollen hier Skateboarder*innen als Köder für die kreative Klasse fungieren, können aber auch als Teil dieser angesehen werden. 


\section{Informeller Urbanismus und Kreativität als Standortfaktor in der Stadt}

Eine genauere Betrachtung des Spannungsfeldes zwischen informellen Urbanismen und der „Creative City“ nimmt der Londoner Geograf Oli Mould vor. Er steht dem Konzept, wie übrigens mittlerweile Richard Florida selbst (vgl. Florida 2017), kritisch gegenüber und geht detailliert auf die Entstehungsgeschichte und Kritiken des Creative-City-Paradigmas ein. Schlussendlich setzt er den Stadtentwicklungsprozessen der „Creative City“ die „urban subversion“ bzw. die „,creative city“ (mit kleinen Anfangsbuchstaben) entgegen. Unter ersterem Begriff fasst Mould (2015: 4) kreative Praktiken wie Graffiti, Parkour, Skateboarding, StreetArt oder Urban Exploration zusammen - also im Grunde jene urbanen Subkulturen, welche auch in der Sparte der informellen Urbanismen genannt werden. „Urban subversion“ ist allerdings kein direktes Synonym für die urbanen Subkulturen. Vielmehr spricht Mould von , those urban social formations that are temporary, fluid and fleeting the momentary crystallisation of creative practices that reappropriate the urban topology in innovate and unexpected ways " (Mould 2015: 115).

Als zentralen Bestandteil davon sieht Mould das ständige Bestreben, auf neue Arten mit der Stadt zu interagieren. Bänke, Pflanzenkörbe, Fußgängerunterführungen, Zäune und noch viele andere alltägliche Dinge einer Stadt sind in erster Linie für einen bestimmten Zweck entworfen. Wie aber interpretiert ein*e Traceur*in, ein*e Skateboarder*in oder ein*e Trial-Fahrer*in eine Bank? Die Funktion wäre eine andere als die von den Planer*innen zugewiesene (vgl. Kap. 2.1). Sie ist vom System, in dem sie sozusagen gebraucht wird, abhängig. Meist ist dieses System, auf das sich die Funktion des Elements richtet, geprägt vom kapitalistischen Verwertungsdruck (vgl. Mould 2015: 116).

Für Mould bedeutet „urban subversion“, diese neuen Funktionen ausfindig zu machen und sich so einen Schritt vom passiven Konsumenten der Stadt zu entfernen, welcher auf banale Weisen mit der Stadt interagiert. Anstelle dessen bringt das Aufspüren alternativer Funktionen der städtischen Umwelt neue Potenziale zum Vorschein und ist demzufolge wahre Kreativität in der Stadt (ebd.: 119). Des Weiteren stellt Mould die Frage, wie diese wahre Kreativität vor der Vereinnahmung durch die dominanten Kräfte in der Stadt sozusagen gerettet werden könne, wie sie weiterhin als Ursprung für die „creative city“ gesehen werden könne, ohne die Ungleichheiten, sozialen und kulturellen Probleme der „Creative City“ zu reproduzieren. Mould greift dazu auf Deleuze und Guattaris Werk „Capitalism and Schizophrenia“" zurück. Diese interpretieren darin das Begehren neu. Anstelle von Verlangen nach etwas, das nicht vorhanden ist - das Fehlen wird dabei durch soziale Konfiguration produziert -, sehen sie dieses als ein instinktives Verlangen, das die sozialen Konfigurationen zerreißt. Kreativität ist also nicht als Antwort auf 
eine Lücke im System zu sehen, sondern entsteht aus einem instinktiven, verinnerlichten Verlangen, neue Formen zu produzieren (ebd.: 109). Dieses Verlangen wurde auch in Interviewgesprächen mit am Alm-DIY-Projekt beteiligten Personen deutlich: ,Aber sobald es heißt Go! ist der Gedanke auf und davon, und dann bist du in einem Rausch, dann willst du kreativ sein, dann willst du arbeiten, dann willst du einfach deinen Raum, in dem du lebst, selber gestalten und tun " (Interview 1).

Doch dem Aufspüren alternativer und nicht mit unmittelbarem finanziellen Nutzen verbundener Nutzungen der Stadt wird oft repressiv begegnet. Dies zeigt sich in Bezug auf den öffentlichen Raum und Skateboarden vor allem in Verboten, durchgesetzt mittels defensiver Architektur oder Sicherheitsdiensten (vgl. Chiu 2009). Im Falle der ersten Bebauung auf dem Nordbahnhofgelände kam es zum Abbruch des ersten in Eigenregie ausgeführten Aufbaus der Rampen. Auch wenn dies nicht direkt im Zuge einer Creative City Policy geschah, war es doch eine Folge des sich durchsetzenden Hegemonialsystems in der Stadt.

Trotzdem ist Skateboarden schon länger in einem Prozess einer „Subculturalisation" (Mould 2015) angekommen. Ausdruck davon sind u. a. die Aufnahme als Sportart bei den Olympischen Spielen oder Sponsoren- und Werbeverträge von Skateboarder*innen mit großen Konzernen (vgl. Schweer 2014) sowie auf räumlicher Ebene die Skateparks oder Skateplazas (vgl. Kap. 2.2), welche entlang der folgenden Beschreibung betrachtet werden können:

„Then, homogenization forces proceed to formalize them (urban social formations, Anm.) and incorporate them as part of dominant practices by re-branding them as cultural products subjugated to commercialization processes within a rationally bounded (and tightly regulated) urban landscape (the process of 'accruing') "(Daskalaki \& Mould 2013: 2).

Neben dieser Tendenz, Skateboarde*innen in regulierte Räume zu fassen, gibt es in der Skateboardszene das Bestreben, sich der Kommodifizierung zu entziehen. Hier ist auch die Praxis des DIY-Skatens zu verorten. In diesem Versuch, der Vereinnahmung zu entfliehen, liegt laut Mould (2015: 167) das Potenzial für urbane Subversion. Umgelegt auf die verschiedenen Praktiken des Skateboardfahrens finden sich diese Komponenten vor allem beim DIY- und beim Street-Skaten wieder (vgl. Kap. $2.1 \& 2.3$ ).

Eine Betrachtung der Entwicklungsgeschichte des Spots „Alm DIY“ erscheint in diesem Spannungsfeld als besonders interessant. Der DIY-Spot auf dem Nordbahnhofgelände ist in seinem Charakter als einstiger „,dead space“, der für seinen subkulturellen Nutzen wiederangeeignet wurde, als Stätte einer ,urban subversion“ zu sehen (vgl. Mould 2015: 139). Im Vergleich zum Skatepark- oder Plaza-Skaten ist das DIY-Skaten aber im Grunde der für die „urban subversion“ 
essenziellen Abkehr vom Mainstream zuzuordnen (vgl. Peters 2016: 283). Schlussendlich wurde das Projekt dort aber beendet - es fand also eine Marginalisierung der subversiven Praxis des DIY-Skatens und der entstandenen Zwischennutzung statt. Der Umzug an die neue Location in St. Marx zeigt, wie in ihrem Charakter der „urban subversion“ zuordenbare Projekte kooptiert werden können. In diesem Fall geschah dies auch explizit unter einer der „Creative City“ zuordenbaren Stadtentwicklungspolitik - mit dem Zweck, die „creative class“ (Florida 2002) anzuwerben.

Wenn man so will, schafft es die „Creative City“ im Geiste des „,new spirit of capitalism“ (Boltanski \& Chiapello 2005) die dem Alm-DIY-Projekt innewohnende subversive Kritik am Kapitalismus durch Kooption zu nehmen. Dass der DIY-Spot nur als Zwischennutzung konzipiert ist und in dem als „space for the industries of tomorrow - for life sciences, for media and the creative professions, and for technology" (WSE 2016: 8) entwickelten Viertel sehr wahrscheinlich keinen Platz mehr haben wird, weist in dieser Hinsicht Parallelen zu dem von Howell (2005) behandelten Beispiel des John F. Kennedy Plaza (auch bekannt als Love Park) in Philadelphia, USA, auf:

„In recent years, the youthful defiance of skateboarders has serendipitously reclaimed Love Park - generating revenue through media exposure and producing a marketable image for the city - all while deterring the presence of homeless. Now the skateboarders, in turn, are being phased out to make way for the next reclamation " (Howell 2005: 41).

Beim neuen DIY-Projekt in St. Marx befinden sich die Skateboarder*innen teilweise in einer ähnlichen Rolle. Die Anerkennung für das dem „bohemian index“ zuträgliche Auftreten der Skater*innen (vgl. Howell 2005: 38) sorgt für den Background, um diese als Werbeträger*innen für den Standort neu marx einzuladen. Gleichzeitig aber ist deren eigene Verdrängung absehbar, da Skateboarden - zumindest jenes in nicht explizit dafür vorgesehenen Flächen - nach der Beendigung der Zwischennutzung und der Fertigstellung des Viertels wohl dem in den physischen Manifestationen der „Creative City“ beobachtbaren ,increase in securitisation and privatisation of the urban realm" (Mould 2015: 85 f.) zum Opfer fallen wird. Für Skateboarder*innen selbst ist eine zur Verfügung gestellte Fläche, auf welcher sie zumindest vorübergehend ihre Vorstellung eines DIY-Skateparks verwirklichen können, aber eine positive Sache. „, To be given a piece of land where skaters can build whatever they want with a small budget, each year they will add sections. An organic social sculpture - super sick" (Pontus Alv zit. nach Irvine 2013: o. S.). Aus dieser Perspektive betrachtet, kann das Zurverfügungstellen der Fläche zumindest vorübergehend als Win-win-Situation betrachtet werden. 
Besonders interessant am Beispiel neu marx ist, dass nicht, wie vielleicht zu erwarten wäre, ein Skatepark oder eine Skateplaza, sondern die gerade als Gegenströmung zur Kommodifizierung verstandene Praxis des DIY-Skatens für die Bewerbung des Ortes aufgegriffen wird. Eine Erklärung bietet die von Miles beschriebene Charakteristik für ,culturally-led urban strategies“: „In its branded, symbolic economy, even street-life and contestations of space are re-packaged" (Miles 2013: 6).

\section{$7 \quad$ Fazit}

Bei der Betrachtung des Alm-DIY-Projekts stellte sich die Frage nach der Positionierung und Einordnung im Spannungsfeld zwischen den Attributen, die mit dem Begriff des „Rechts auf Stadt" verbunden sind, und der Frage, inwiefern diese das Projekt wiederum für eine an die „Creative City“ angelehnte Stadtentwicklungsstrategie verwertbar machen. Die analysierte Zwischennutzung bewegte sich zunehmend weg von einer wilden Raumaneignung und hin zu einem gezielt eingesetzten Faktor für die Standortbewerbung eines im Entstehen befindlichen Viertels für die Kreativwirtschaft. Der Anspruch, die Stadt und deren Freiräume als Bewohner*innen der Stadt auch selbst gestalten zu können, war vor allem am ersten Standort des Projekts deutlich erkennbar. Zusammen mit der vielfältigen Nutzung und der Einbettung im Quartier zeigte das Projekt so Alternativen für eine demokratischere Stadt von unten auf. Die Initiative fand auch Beachtung und Förderung vonseiten der städtischen Planung. Trotzdem konnte der organisch gewachsene Freiraum auf dem Nordbahnhofgelände mittelfristig nicht gesichert werden. Ein erneuter Aufbau wurde durch die Anwerbung der Gruppe für eine Zwischennutzung auf einer anderen Brachfläche ermöglicht, dieses Mal jedoch gekoppelt an die Erwartungshaltung, das als Standort für Medien, Forschung und Technologie konzipierte Quartier zu bewerben.

\section{Literatur}

Boltanski, L. \& Chiapello, E. (2005): The New Spirit of Capitalism. London. Borden, I. (2001): Skateboarding, Space and the City: Architecture and the Body. Oxford. Chiu, C. (2009): Contestation and Conformity. Street and Park Skateboarding in New York City Public Space. Space and Culture (12) 1: 25-42.

Daskalaki, M., \& Mould, O. (2013): Beyond Urban Subcultures: Urban Subversions as Rhizomatic Social Formations. International Journal of Urban and Regional Research (37) $1: 1-18$.

Florida, R. (2002). The Rise Of The Creative Class. And How It's Transforming Work, Leisure, Community and Everyday Life. New York. 
Florida, R. (2017): The New Urban Crisis: How Our Cities Are Increasing Inequality, Deepening Segregation, and Failing the Middle Class - and What We Can Do About It. New York.

Freivogelheit GmbH (2012): Vogelfreiheit. Grün Berlin. Abrufbar unter: https://gruen-berlin.de/file/2126/download?token=QYCRMDL0. Letzter Zugriff: 24.4.2018.

Gebhardt, D. \& Holm, A. (2011): Initiativen für ein Recht auf Stadt. In: Holm, A. \& Gebhardt, D. (Hrsg.): Initiativen für ein Recht auf Stadt. Theorie und Praxis städtischer Aneignung. Hamburg: 7-25.

Grabner, D. (2016): ALM DIY vor dem Aus? Radio FM4. Abrufbar unter: http://fm4. orf.at/stories/1768219/. Letzter Zugriff: 24.4.2018.

Hahn, A. (2016): LOVE Park renovation breaks ground, skateboarding ban temporarily lifted. PlanPhilly. Abrufbar unter: http://planphilly.com/articles/2016/02/10/love-park-renovation-breaks-ground-skateboarding-ban-temporarily-lifted. Letzter Zugriff: 24.4.2018.

Howell, O. (2005): The „Creative Class“ and the Gentrifying City. Skateboarding in Philadelphia's Love Park. Journal of Architectural Education (59) 2: 32-42.

Howell, O. (2008): Skatepark as Neoliberal Playground. Space and Culture (11) 4: 475-496.

Ihaza, J. (2018): Skateboarders Won. The New York Times. www.nytimes.com/2018/ 04/07/style/skateparks-around-the-world.html. Letzter Zugriff: 24.4.2018.

Irvine, A. (2013): Pontus Alv's DIY interview from issue 98. Kingpinmag. Abrufbar unter: https://kingpinmag.com/features/pontus-alvs-diy-interview-from-issue- $98 . \mathrm{html}$. Letzter Zugriff: 24.4.2018.

Iveson, K. (2013): Cities within the City: Do-It-Yourself Urbanism and the Right to the City. International Journal of Urban and Regional Research (37) 3: 941-956.

Kingsford, H. (2013): Jacob Harris Interview. Grey Skate Mag. http://www.greyskatemag. com/2013/10/jacob-harris-interview/. Letzter Zugriff: 24.4.2018.

Lombard, K. (2010): Skate and create/skate and destroy: The commercial and governmental incorporation of skateboarding. Continuum (24) 4: 475-488.

Magistratsabteilung 21 (2015) (Hrsg.): Handbuch zum städtebaulichen Leitbild Nordbahnhof. https://www.wien.gv.at/stadtentwicklung/projekte/nordbahnhof/grundlagen/leitbild-2014/pdf/handbuch-gesamt.pdf. Letzter Zugriff: 24.4.2018.

Marcuse, P. (2009): From critical urban theory to the right to the city. Cities (13) 2-3: 185-197.

Mayer, M. (2011): Recht auf die Stadt - Bewegungen in historisch und räumlich vergleichender Perspektive. In: Holm, A. \& Gebhardt, D. (Hrsg.): Initiativen für ein Recht auf Stadt. Theorie und Praxis städtischer Aneignung. Hamburg: 53-79.

Mayer, M. (2013): Urbane soziale Bewegungen in der neoliberalisierenden Stadt. sublurban Zeitschrift für Kritische Stadtforschung (1) 1: 155-168.

Miles, M. (2013): A Post-Creative City? RCCS Annual Review (5) 5.

Mould, O. (2015): Urban Subversion and the Creative City. New York.

Mullis, D. (2013): Recht auf die Stadt. Facetten und Möglichkeiten einer Parole. Emanzipation (3) 2: 57-70.

Peters, C. (2016): Skateboarding. Ethnographie einer urbanen Praxis. Münster.

Purcell, M. (2014): Possible Worlds: Henri Lefebvre and the Right to the City. Journal of Urban Affairs (36) 1: 141-154. 
Raumposition. (2016): Neu Marx gemeinsam gestalten. Aufgabenstellung für die Entwicklung von Neu Marx. Abrufbar unter: www.raumposition.at/download/neumarx/01 NMX_Aufgabenstellung.pdf. Letzter Zugriff: 24.4. 2018.

Schweer, S. (2014): Skateboarding. Zwischen urbaner Rebellion und neoliberalem Selbstentwurf. Bielefeld.

Vivoni, F. (2013): Waxing ledges: built environments, alternative sustainability, and the Chicago skateboarding scene. Local Environment (18) 3: 340-353.

Willinger, S. (2014): Governance des Informellen. Informationen zur Raumentwicklung (2014) 2: 147-156.

WSE Wiener Standort Entwicklungs GmbH (2016): Fact Sheet Neu Marx - Hier passiert Zukunft. Abrufbar unter: http://www.neumarx.at/tools/uploads/Factsheet-Gesamtareal2016.pdf. Letzter Zugriff: 24.4.2018.

Open Access Dieses Kapitel wird unter der Creative Commons Namensnennung 4.0 International Lizenz (http://creativecommons.org/licenses/by/4.0/deed.de) veröffentlicht, welche die Nutzung, Vervielfältigung, Bearbeitung, Verbreitung und Wiedergabe in jeglichem Medium und Format erlaubt, sofern Sie den/die ursprünglichen Autor(en) und die Quelle ordnungsgemäß nennen, einen Link zur Creative Commons Lizenz beifügen und angeben, ob Änderungen vorgenommen wurden.

Die in diesem Kapitel enthaltenen Bilder und sonstiges Drittmaterial unterliegen ebenfalls der genannten Creative Commons Lizenz, sofern sich aus der Abbildungslegende nichts anderes ergibt. Sofern das betreffende Material nicht unter der genannten Creative Commons Lizenz steht und die betreffende Handlung nicht nach gesetzlichen Vorschriften erlaubt ist, ist für die oben aufgeführten Weiterverwendungen des Materials die Einwilligung des jeweiligen Rechteinhabers einzuholen.

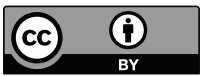

